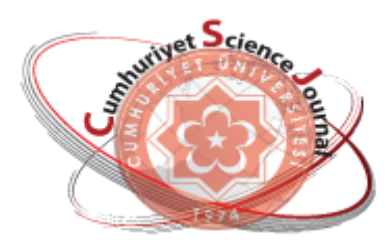

e-ISSN: $2587-246 X$

ISSN: $2587-2680$

\title{
Ambarzumyan Type Theorems for a Class of Sturm-Liouville Problem
}

\author{
A. Sinan OZKAN ${ }^{1}$, Yasar CAKMAK ${ }^{1 *}$ \\ ${ }^{1}$ Cumhuriyet University, Faculty of Science, Department of Mathematics, 58140 Sivas, Turkey
}

\begin{abstract}
In this paper, we prove Ambarzumyan type theorems for an impulsive Sturm-Liouville problem with eigenparameter in the boundary conditions.
\end{abstract}

Keywords: Ambarzumyan theorem, Sturm-Liouville equation, Inverse problem.

\section{Bir Sınıf Sturm-Liouville Problemi için Ambarzumyan Tipi Teoremler}

Özet: Bu makalede, sınır koşulları parametreye bağlı, bir geçiş koşullu Sturm-Liouville problemi için Ambarzumyan tipi teoremler ispatlanmaktadır.

Anahtar Kelimeler: Ambarzumyan teoremi, Sturm-Liouville denklemi, Ters problem.

\section{INTRODUCTION}

Inverse spectral problems consist in recovering the coefficients of an operator from their spectral characteristics. The first study which started inverse spectral theory for SturmLiouville operator was investigated by Ambarzumyan [1] in 1929. He proved that if $q(x)$ is continuous function on $(0,1)$ and the eigenvalues of the problem

$$
\left\{\begin{array}{l}
-y^{\prime \prime}+q(x) y=\lambda y, x \in(0,1) \\
y^{\prime}(0)=y^{\prime}(1)=0
\end{array}\right.
$$

are given as $\lambda_{n}=n^{2} \pi^{2}, n \geq 0$, then $q(x) \equiv 0$.

We refer to some Ambarzumyan type theorems for the Sturm-Liouville and Dirac operators in [2]-[11].
Particularly, in [2], an extension of Ambarzumyan's theorem is given for SturmLiouville problem with general boundary conditions. In [3], the classical Ambarzumyan's theorem is proven for the regular SturmLiouville problem with the eigenvalue parameter in the boundary conditions. In [4], some particular generalizations of the classical Ambarzumyan theorem are proven for the regular Sturm-Liouville problem with the discontinuity conditions.

The aim of this paper is to prove two Ambarzumyan type theorems for the impulsive Sturm-Liouville problem with the eigenvalue parameter in one boundary condition. 


\section{Preliminaries:}

We consider the boundary value problem $L=L\left(q, \frac{a}{b}, \alpha_{1}, \alpha_{2}\right)$ generated by the regular

Sturm-Liouville equation

$$
-y^{\prime \prime}+q(x) y=\lambda y, x \in(0,1)
$$

subject to the boundary conditions

$$
\begin{aligned}
& y^{\prime}(0)=0 \\
& a(\lambda) y(1)+b(\lambda) y^{\prime}(1)=0
\end{aligned}
$$

and the discontinuity conditions

$$
\left\{\begin{array}{c}
y\left(\frac{1}{2}+0\right)=\alpha_{1} y\left(\frac{1}{2}-0\right) \\
y^{\prime}\left(\frac{1}{2}+0\right)=\alpha_{2} y^{\prime}\left(\frac{1}{2}-0\right)
\end{array}\right.
$$

where $\lambda$ is the spectral parameter; $q(x)$ is a continuous function on $(0,1) ; \alpha_{1}, \alpha_{2} \in R-\{1\}$ and for $a_{k}, b_{k} \in R, a_{m} \neq 0, b_{m}=1, m \in Z^{+}$

$$
a(\lambda)=\sum_{k=1}^{m} a_{k} \lambda^{k}, \quad b(\lambda)=\sum_{k=0}^{m} b_{k} \lambda^{k} .
$$

Let us denote a solution of (1.1) by $\varphi(x, \lambda)$ satisfying the initial conditions

$$
\varphi(0, \lambda)=1, \varphi^{\prime}(0, \lambda)=0
$$

and the discontinuity conditions (1.4).

The following asymptotics are given in [12]:

$$
\begin{aligned}
& \varphi(x, \lambda)=\cos \sqrt{\lambda} x+\omega(x) \frac{\sin \sqrt{\lambda} x}{\sqrt{\lambda}} \\
& +o\left(\frac{1}{\sqrt{\lambda}} \exp |\tau| x\right), \quad x<\frac{1}{2}
\end{aligned}
$$

$$
\begin{aligned}
& \varphi(x, \lambda)=\alpha^{+} \cos \sqrt{\lambda} x \\
& +\alpha^{-} \cos \sqrt{\lambda}(1-x) \\
& +\alpha^{+} \omega(x) \frac{\sin \sqrt{\lambda} x}{\sqrt{\lambda}} \\
& -\alpha^{-}\left(\omega\left(\frac{1}{2}\right)-\omega(x)\right) \frac{\sin \sqrt{\lambda}(1-x)}{\sqrt{\lambda}} \\
& +o\left(\frac{1}{\sqrt{\lambda}} \exp |\tau| x\right), \quad x>\frac{1}{2}
\end{aligned}
$$

and

$\varphi^{\prime}(x, \lambda)=-\sqrt{\lambda} \sin \sqrt{\lambda} x$

$+\omega(x) \cos \sqrt{\lambda} x+o(\exp |\tau| x), \quad x<\frac{1}{2}$

$$
\begin{aligned}
& \varphi^{\prime}(x, \lambda)=-\sqrt{\lambda} \alpha^{+} \sin \sqrt{\lambda} x \\
& +\sqrt{\lambda} \alpha^{-} \sin \sqrt{\lambda}(1-x) \\
& +\alpha^{+} \omega(x) \cos \sqrt{\lambda} x \\
& -\alpha^{-}\left(\omega\left(\frac{1}{2}\right)-\omega(x)\right) \cos \sqrt{\lambda}(1-x) \\
& +o(\exp |\tau| x), \quad x>\frac{1}{2}
\end{aligned}
$$

where $\quad \omega(x)=\frac{1}{2} \int_{0}^{x} q(t) d t, \quad \alpha^{\mp}=\frac{1}{2}\left(\alpha_{1} \mp \alpha_{2}\right)$,

$$
\tau=\operatorname{Im} \sqrt{\lambda}
$$

The function

$$
\Delta(\lambda):=a(\lambda) \varphi(1, \lambda)+b(\lambda) \varphi^{\prime}(1, \lambda)
$$

is entire on $\lambda$ and the roots of $\Delta(\lambda)=0$ are coincide with eigenvalues of the problem $L$.

From (1.8), (1.10) and (1.11), we have

$$
\begin{aligned}
& \Delta(\lambda)=-\alpha^{+} \lambda^{m}\{\sqrt{\lambda} \sin \sqrt{\lambda} \\
& -\left(\omega(1)+a_{m}\right) \cos \sqrt{\lambda} \\
& \left.+\frac{\alpha^{-}}{\alpha^{+}}\left(\omega\left(\frac{1}{2}\right)-\omega(1)-a_{m}\right)+o(\exp |\tau|)\right\}
\end{aligned}
$$


Let $\sigma(L)=\left\{\lambda_{n}\right\}_{n \geq 0}$ be the set of the eigenvalues of $L$. The numbers $\lambda_{n}$ satisfy the following asymptotic formula for $n \rightarrow \infty$ :

$$
\begin{aligned}
& \lambda_{n}=(n-m) \pi+\frac{1}{(n-m) \pi}\left\{\omega(1)+a_{m}\right. \\
& \left.+(-1)^{n-m} \frac{\alpha^{-}}{\alpha^{+}}\left(\omega\left(\frac{1}{2}\right)-\omega(1)-a_{m}\right)\right\} \\
& +o\left(\frac{1}{n}\right) .
\end{aligned}
$$

\section{Main Results:}

We consider the problem $L_{0}=L\left(0, \frac{a}{b}, \alpha_{1}, \alpha_{2}\right)$ together with $L$. It is obvious that eigenvalues of the problem $L_{0}$ satisfy the following asymptotic relation for $n \rightarrow \infty$

$$
\begin{aligned}
& \lambda_{n}^{0}=(n-m) \pi \\
& +\frac{1}{(n-m) \pi}\left\{a_{m}-(-1)^{n-m} \frac{\alpha^{-}}{\alpha^{+}} a_{m}\right\} \\
& +o\left(\frac{1}{n}\right) .
\end{aligned}
$$

Lemma 1 If $\lambda_{n}=\lambda_{n}^{0}$ for sufficiently large $n$, then $\int_{0}^{1} q(x) d x=0$.

Proof. If $\lambda_{n}=\lambda_{n}^{0}$ as $n \rightarrow \infty$, then

$$
\begin{aligned}
& (n-m) \pi+\frac{1}{(n-m) \pi}\left\{\omega(1)+a_{m}\right. \\
& \left.+(-1)^{n-m} \frac{\alpha^{-}}{\alpha^{+}}\left(\omega\left(\frac{1}{2}\right)-\omega(1)-a_{m}\right)\right\}+o\left(\frac{1}{n}\right) \\
& =(n-m) \pi \\
& +\frac{1}{(n-m) \pi}\left\{a_{m}-(-1)^{n-m} \frac{\alpha^{-}}{\alpha^{+}} a_{m}\right\}+o\left(\frac{1}{n}\right)
\end{aligned}
$$

and so

$$
\omega(1)+(-1)^{n-m} \frac{\alpha^{-}}{\alpha^{+}}\left(\omega\left(\frac{1}{2}\right)-\omega(1)-a_{m}\right)=o(1)
$$

for sufficiently large $n$. Therefore, we get

$$
\left\{\begin{array}{l}
\omega(1)+\frac{\alpha^{-}}{\alpha^{+}}\left(\omega\left(\frac{1}{2}\right)-\omega(1)-a_{m}\right)=0 \\
\omega(1)-\frac{\alpha^{-}}{\alpha^{+}}\left(\omega\left(\frac{1}{2}\right)-\omega(1)-a_{m}\right)=0
\end{array} .\right.
$$

Thus $\omega(1)=0$ i.e. $\int_{0}^{1} q(x) d x=0$.

\section{Theorem 1 If}

$\left\{(n-m) \pi+\frac{a_{m}}{(n-m) \pi}+o\left(\frac{1}{n}\right): n>n_{0}\right\} \cup\{0\} \subset \sigma(L)$

for some $n_{0} \in \mathrm{N}$, then $q(x) \equiv 0$ a.e. on $(0,1)$.

Proof. From Lemma 1, it is obtained that $\int_{0}^{1} q(x) d x \equiv 0 . \quad$ On the other hand, since $0 \in \sigma(L)$, we get $q(x) \equiv 0$ a.e. on $(0,1)$ from the classical Ambarzumyan theorem.

Theorem 2 If $\lambda_{s}$ is an eigenvalue of the problem $L$ such that $b\left(\lambda_{s}\right) \neq 0$ and $\int_{0}^{1} q(x) d x-\lambda_{s}+\frac{a\left(\lambda_{s}\right)}{b\left(\lambda_{s}\right)}=0$, then $q(x) \equiv \lambda_{s}$, a.e. on $(0,1)$ and $a\left(\lambda_{s}\right)=0$.

Proof. Let $y_{s}(x)$ be the eigenfunction corresponding to $\lambda_{s}$. Then we can write for $x \in(0,1)$

$$
\left\{\begin{array}{l}
-y_{s}^{\prime \prime}(x)+q(x) y_{s}(x)=\lambda_{s} y_{s}(x) \\
y_{s}^{\prime}(0)=0 \\
a\left(\lambda_{s}\right) y_{s}(1)+b\left(\lambda_{s}\right) y_{s}^{\prime}(1)=0
\end{array}\right.
$$

It is clear that $y_{s}(0) \neq 0$ and $y_{s}(1) \neq 0$. Otherwise, since $b\left(\lambda_{s}\right) \neq 0, \quad y_{s}^{\prime}(0)=0$ or 
$y_{s}^{\prime}(1)=0$. In both cases, $y_{s}(x) \equiv 0$ by the uniqueness of the solution of an initial value problem.

The function $y_{s}(x)$ has finitely many isolated nodes on $(0,1)$ and $y_{s}\left(x_{i}\right)=0$ yields $y_{s}^{\prime \prime}\left(x_{i}\right)=0$ but $y_{s}^{\prime}\left(x_{i}\right) \neq 0$. Then the function $\frac{y_{s}^{\prime \prime}(x)}{y_{s}(x)}$ is bounded in the neighborhood of each $x_{i}$.

From (2.2) and the relation $\frac{y_{s}^{\prime \prime}(x)}{y_{s}(x)}=\left(\frac{y_{s}^{\prime}(x)}{y_{s}(x)}\right)^{\prime}+\left(\frac{y_{s}^{\prime}(x)}{y_{s}(x)}\right)^{2}, \quad$ we get $\left(\frac{y_{s}^{\prime}(x)}{y_{s}(x)}\right)^{\prime}=q(x)-\lambda_{s}-\left(\frac{y_{s}^{\prime}(x)}{y_{s}(x)}\right)^{2}$.

By integrating of both sides from 0 to 1 , the following equality is obtained

$$
\int_{0}^{1}\left(\frac{y_{s}^{\prime}(x)}{y_{s}(x)}\right)^{2} d x=\frac{a\left(\lambda_{s}\right)}{b\left(\lambda_{s}\right)}+\int_{0}^{1} q(x) d x-\lambda_{s}=0 .
$$

Thus $y_{s}^{\prime}(x) \equiv 0$ and so $y_{s}(x) \equiv$ constant. Hence, it is concluded from (2.2) that $q(x)=\lambda_{s}$ a.e. on $(0,1)$ and $a\left(\lambda_{s}\right)=0$.

\section{REFERENCES}

[1]. Ambartsumyan, V. A., Uber eine frage der eigenwerttheorie, Zeitschrift für Physik, 1929, 53, 690-695.

[2]. Chern, H. H., Law, C. K., Wang, H.J., Corrigendum to extension of Ambarzumyan's theorem to general boundary conditions, J. Math. Anal. Appl., 2005, 309, 764-768.

[3]. Yang, C. F. and Yang, X. P., Ambarzumyan's theorem with eigenparameter in the boundary conditions, Acta Math. Sci., 2011, 31B(4), 1561-1568.
[4]. Yurko, V. A., On Ambarzumyan-type theorems, Applied Math. Letters, 2013, 26, 506-509.

[5]. Yang, C. F. and Yang, X. P., Ambarzumyan's theorems for SturmLiouville operators with general boundary conditions, Acta Math. Sci., 2010, 30A(2), 449-455.

[6]. Horváth, M., On a theorem of Ambarzumyan, Proc. Roy. Soc.Edinburgh Sect. A, 2001, 131(4), 899-907.

[7]. Yang, C. F. and Yang, X. P., Some Ambarzumyan-type theorems for Dirac operators, Inverse Problems, 2009, 25(9), 095012pp.13.

[8]. Chern, H. H. and Shen, C. L., On the ndimensional Ambarzumyan's theorem, Inverse Problems, 1997, 13, 15-18.

[9]. Márton, K., An n-dimensional Ambarzumyan type theorem for Dirac operators, Inverse Problems, 2004, 20, 1593-1597.

[10]. Yang, C. F., Huang, Z. Y. and Yang, X. P., Ambarzumyan's theorems for vectorial Sturm-Liouville systems with coupled boundary conditions, Taiwanese J. Math., 2010, 14(4), 14291437.

[11]. Kırac, A. A., On the Ambarzumyan's theorem for the quasi-periodic problem, Analysis and Math. Physics, 2015, 13, 15-18.

[12]. Freiling, G. and Yurko, V. A., Inverse Sturm-Liouville problems and their applications, Nova Science, Huntington, $N Y, 2001$ 